ARTICLE

https://doi.org/10.1038/s41467-020-18521-6

\title{
OPEN
}

\section{Probing magnetism in atomically thin semiconducting $\mathrm{PtSe}_{2}$}

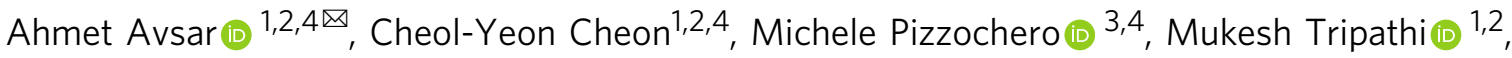 \\ Alberto Ciarrocchi (1) ${ }^{1,2}$, Oleg V. Yazyev (i) ${ }^{3}$ \& Andras Kis (1) ${ }^{1,2}$.
}

Atomic-scale disorder in two-dimensional transition metal dichalcogenides is often accompanied by local magnetic moments, which can conceivably induce long-range magnetic ordering into intrinsically non-magnetic materials. Here, we demonstrate the signature of long-range magnetic orderings in defective mono- and bi-layer semiconducting $\mathrm{PtSe}_{2}$ by performing magnetoresistance measurements under both lateral and vertical measurement configurations. As the material is thinned down from bi- to mono-layer thickness, we observe a ferromagnetic-to-antiferromagnetic crossover, a behavior which is opposite to the one observed in the prototypical 2D magnet $\mathrm{Crl}_{3}$. Our first-principles calculations, supported by aberration-corrected transmission electron microscopy imaging of point defects, associate this transition to the interplay between the defect-induced magnetism and the interlayer interactions in $\mathrm{PtSe}_{2}$. Furthermore, we show that graphene can be effectively used to probe the magnetization of adjacent semiconducting $\mathrm{PtSe}_{2}$. Our findings in an ultimately scaled monolayer system lay the foundation for atom-by-atom engineering of magnetism in otherwise non-magnetic 2D materials.

\footnotetext{
${ }^{1}$ Electrical Engineering Institute, École Polytechnique Fédérale de Lausanne (EPFL), Lausanne 1015, Switzerland. ${ }^{2}$ Institute of Materials Science and Engineering, École Polytechnique Fédérale de Lausanne (EPFL), Lausanne, CH 1015, Switzerland. ${ }^{3}$ Institute of Physics, École Polytechnique Fédérale de Lausanne (EPFL), Lausanne, CH 1015, Switzerland. ${ }^{4}$ These authors contributed equally: Ahmet Avsar, Cheol-Yeon Cheon, Michele Pizzochero.

凶email: ahmet.avsar@epfl.ch; andras.kis@epfl.ch
} 


\section{T} wo-dimensional (2D) transition metal dichalcogenides $(\mathrm{TMDCs})^{1}$ are emerging as an appealing class of materials for a wide range of research topics ${ }^{1}$, including electronics ${ }^{2}$, spintronics $^{3-6}$, and magnetism ${ }^{7-10}$. Among this rapidly expanding family of materials, layered platinum diselenide $\left(\mathrm{PtSe}_{2}\right)$ is a relatively new and interesting member due to its unique thickness-dependent electronic structure ${ }^{11-17}$. While multilayer films exhibit metallic character, this crystal undergoes a metal-tosemiconductor transition when thinned down to mono- and bilayers $^{11-13}$. Theoretical work has also indicated that electronic properties of such thin $\mathrm{PtSe}_{2}$ crystal can be further tuned by defect engineering ${ }^{18}$.

Recent experimental investigations highlighted a broad variety of intrinsic point defects in $\mathrm{PtSe}_{2}{ }^{19}$. Even though imperfections are often regarded as detrimental to material properties, in many cases their presence has proven successful in introducing functionalities that would otherwise be absent in pristine materials ${ }^{20,21}$. This is especially true in the case of metallic $\mathrm{PtSe}_{2}$ multilayers, where surface $\mathrm{Pt}$ vacancies trigger a thicknessdependent surface magnetic ordering ${ }^{18,20}$. The strength of the magnetic exchange coupling across the metallic multilayers is governed by the RKKY interactions. However, thin films of $\mathrm{PtSe}_{2}$ acquire a semiconducting character when approaching the singlelayer regime ${ }^{11,13}$, and the physical picture governing the magnetic exchange interactions in the thinnest films of $\mathrm{PtSe}_{2}$ would rather invoke the direct and superexchange mechanisms, similarly to dilute magnetic semiconductors ${ }^{22}$. Understanding and controlling the interplay between disorder and magnetism in this ultimate thickness scale can spark unexplored directions for designing 2D semiconducting magnets through defect engineering $^{8}$. In this Letter, we demonstrate that defect-induced magnetism is preserved in mono and bilayers of semiconducting $\mathrm{PtSe}_{2}$, with a monolayer, surprisingly, showing antiferromagnetic ordering, while bilayer $\mathrm{PtSe}_{2}$ is a ferromagnet.

\section{Results}

Device characterization. Our ultra-thin $\mathrm{PtSe}_{2}$ flakes were obtained by mechanical exfoliation from CVT-grown bulk crystals (HQ Graphene), and their thicknesses were determined by atomic force microscopy (AFM). As shown in Fig. 1a, the thickness of a monolayer (bilayer) is found to be $\sim 0.65 \mathrm{~nm}$ $(\sim 1.2 \mathrm{~nm})$. Next, the mono- or bilayer crystal was transferred onto a monolayer graphene ribbon which was initially deposited onto a $\mathrm{Si} / \mathrm{SiO}_{2}$ substrate. Graphene is utilized as a bottom electrode, thereby taking advantage of its electrostatic transparency. This enables gate-tunable vertical and lateral transport operations, as the screening length exceeds its monolayer thickness ${ }^{23}$. By employing electron-beam lithography and electron-beam evaporation techniques, non-magnetic metallic palladium $(\mathrm{Pd})$ contacts (80-nm thick) were formed on top of $\mathrm{PtSe}_{2}$ and graphene. Further details concerning the device fabrication process are given in "Methods" section. The choice of non-magnetic graphene and $\mathrm{Pd}$ contacts rules out any influence of the electrodes on the magnetic response of the device. The schematics of the resulting device geometry is shown in Fig. 1c. This geometry allows us to probe the magnetism in $\mathrm{PtSe}_{2}$ through vertical transport by passing the charge current between electrodes \#2 (graphene) and \#3 (Pd). In addition, it allows us to characterize the basic transport properties of monolayer $\mathrm{PtSe}_{2}$ by utilizing $\mathrm{Pd}$ electrodes \#3 and \#4 in the lateral measurement geometry. We further compare the magneto-transport properties of isolated graphene as well as the graphene/monolayer $\mathrm{PtSe}_{2}$ heterostructure by relying on electrode pairs \#1 \& \#2 and \#2 \& \#5, respectively. An optical image of the completed device is presented in the bottom panel of Fig. 1c. The transport output was characterized as a function of back-gate voltage $\left(V_{\mathrm{BG}}\right)$, sourcedrain bias $\left(V_{\mathrm{DS}}\right)$, magnetic field, and temperature. Magnetic fielddependent measurements were performed at the base temperature $(1.6 \mathrm{~K})$ to maximize the signal-to-noise ratio.

Charge transport in monolayer $\mathrm{PtSe}_{2}$. Prior to magnetic fielddependent measurements, we first assess the basic charge transport properties of monolayer $\mathrm{PtSe}_{2}$. Figure 1d shows the $V_{\mathrm{BG}}$ dependence of $I_{\mathrm{DS}}$ measured at temperatures covering the $1.6-142 \mathrm{~K}$ range, while maintaining a constant $V_{\mathrm{DS}}=2 \mathrm{~V}$. While $I_{\mathrm{DS}}$ is slightly modulated by the application of $V_{\mathrm{BG}}$ due to the semiconducting nature of monolayer $\mathrm{PtSe}_{2}$, the device shows a relatively low on-state conductance $\left(V_{\mathrm{BG}}>-5 \mathrm{~V}\right)$. The insulating nature of the crystal is unambiguously confirmed by the observed temperature dependence of the conductivity $(\sigma)$ measured at fixed carrier concentrations (Fig. 1e-Inset). In the presence of disorder, the electrical transport can be described using the Mott formalism and the variable range hopping (VRH) model which describes the conductivity $\sigma$ in terms of a characteristic temperature ${ }^{24}$. The observed linear dependence of the $\ln (G)$ on $T^{-1 / 3}$ at different given carrier densities (see Fig. 1e and Supplementary Note 1) supports the 2D VRH nature of charge transport up to room temperature (RT). Such a characteristic temperature dependence has been widely observed in many systems, including defective 2D TMDC monolayers ${ }^{25-27}$. In fact, a bandgap of $1.2 \mathrm{eV}$ is expected in monolayer $\mathrm{PtSe}_{2}{ }^{12}$ which in turn should result in a stable off state over a large $V_{\mathrm{BG}}$ range even at RT. For example, transport characteristics of monolayer black phosophorus ${ }^{28}$ and multilayer $\mathrm{MoSe}_{2}{ }^{29}$, which display comparable bandgap values with monolayer $\mathrm{PtSe}_{2}$, exhibit a stable off-state over $60 \mathrm{~V}$ when measured in a back-gate device geometry with an equivalent $\mathrm{SiO}_{2}$ thickness of $\sim 270 \mathrm{~nm}$. As shown in Fig. 1f, the lack of a clear offstate along with a strong dependence of the off-current on $V_{\mathrm{DS}}$ at RT signals the hopping nature of charge transport, and, importantly, further suggests the presence of atomic defects in monolayer $\mathrm{PtSe}_{2}{ }^{27}$.

Imaging $\mathrm{PtSe}_{2}$ defects using transmission electron microscopy. In order to directly visualize the type of defects in our samples, we relied on Cs-corrected high-resolution transmission electron microscopy imaging. The result obtained from a bilayer $\mathrm{PtSe}_{2}$ with a low accelerating voltage of $80 \mathrm{kV}$ is shown in Fig. 1b. Consistent with early scanning tunneling microscopy investigations $^{19}$, we observe native $\mathrm{Se}$ and $\mathrm{Pt}$ vacancies in ultra-thin $\mathrm{PtSe}_{2}$ films. The concentration of Pt vacancy defects is found to be $\sim 9 \times$ $10^{12} \mathrm{~cm}^{-2}$ (see Supplementary Note 2). Similarly to the bilayer case, we also observe Pt vacancies in monolayers (see Supplementary Note 3).

Magnetoresistance measurement in monolayer $\mathbf{P t S e}_{2}$. After confirming the presence of intrinsic defects in our crystals, we next study the magneto-transport characteristics of our monolayer $\mathrm{PtSe}_{2}$ sandwiched between the source (graphene) and drain (Pd) electrodes (inset of Fig. 2a) by utilizing a vertical measurement geometry. A fixed $V_{\mathrm{DS}}$ is applied to $\mathrm{PtSe}_{2}$ while measuring the dependence of device resistance on the applied magnetic field. As shown in Fig. 2a, the $I_{\mathrm{DS}}-V_{\mathrm{DS}}$ curve is nearly linear and symmetric, measured at zero field. As compared to the lateral transport measurement shown in Fig. 1d, the device resistance under vertical geometry is improved by several orders of magnitude, as a result of the reduced transport pathway. Such a highly conductive response obtained along the vertical direction allow$s$ us to probe directly the magnetic ordering of monolayer $\mathrm{PtSe}_{2}$. To this end, we sweep the out-of-plane magnetic field from -0.2 to $0.2 \mathrm{~T}$ while recording the vertical device resistance at 
a
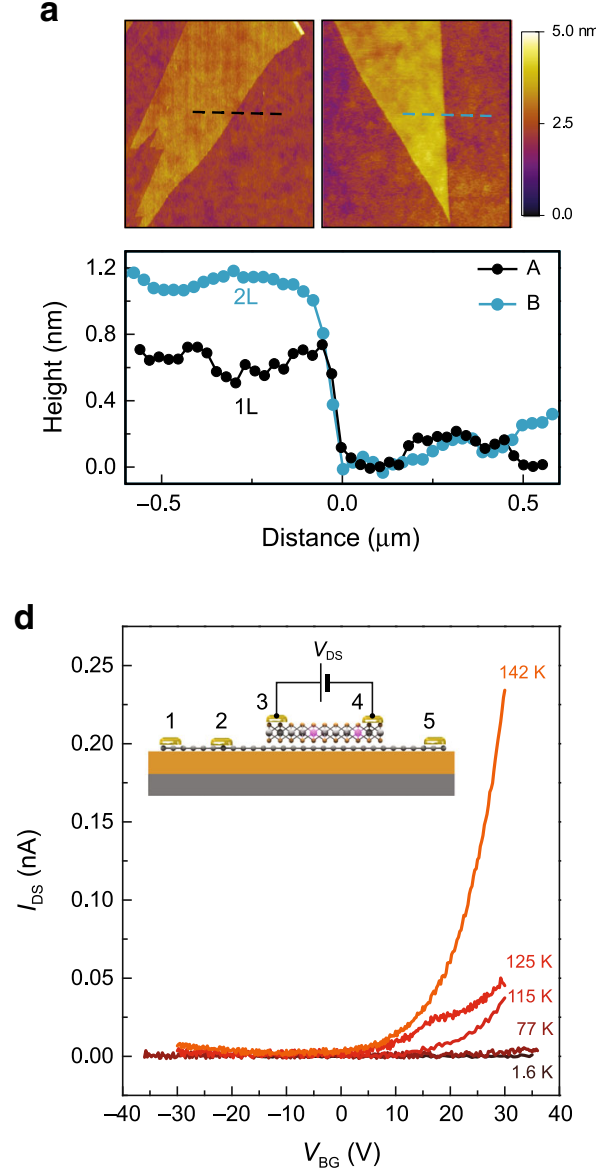

b

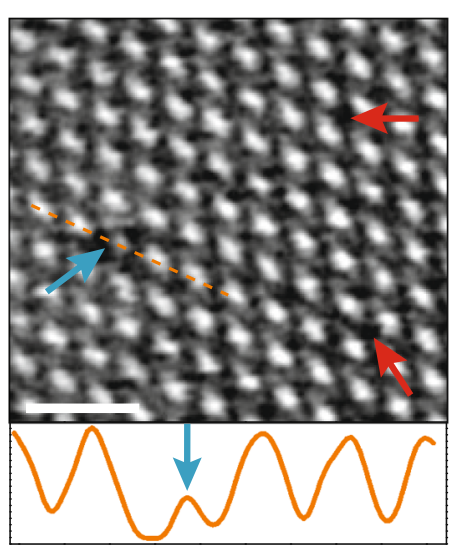

C

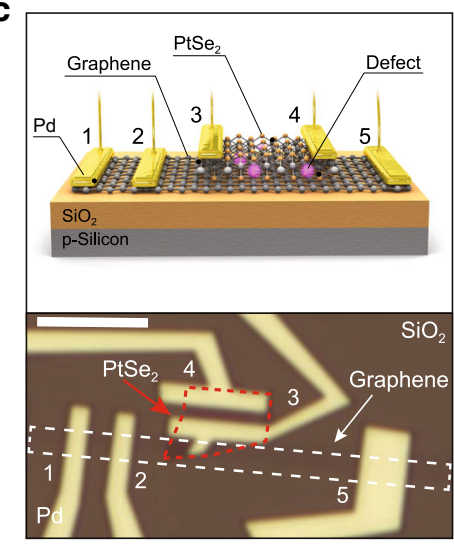

e

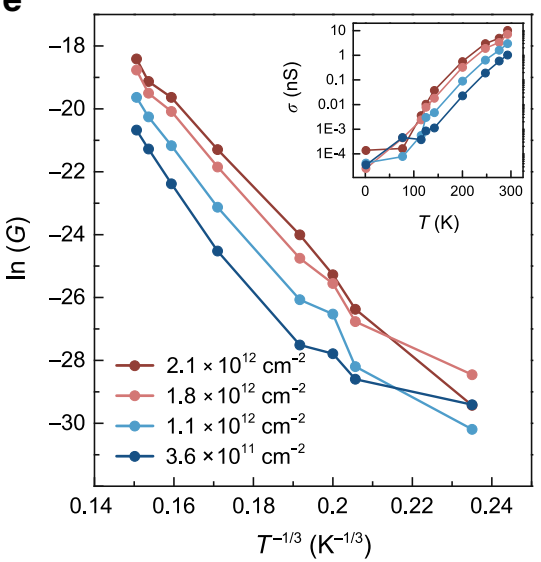

f

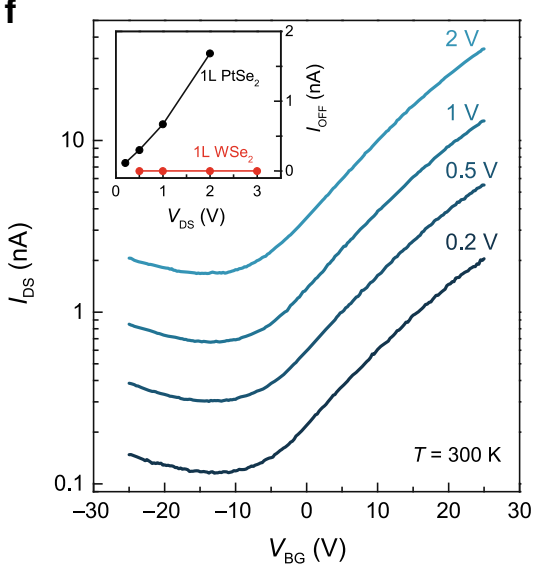

Fig. 1 Device structure and basic characterization. a AFM images for mono- and bilayer $\mathrm{PtSe}_{2}$ which are utilized as active channel materials in characterized devices. Color scale of the AFM image is $0-5 \mathrm{~nm}$. Following the dashed lines, a height of $\sim 0.65 \mathrm{~nm}(\sim 1.2 \mathrm{~nm})$ is measured for monolayer (bilayer) PtSe $e_{2}$ Scan size: $\sim 3 \mu \mathrm{m} \times 3 \mu \mathrm{m}$. b Raw HAADF image of a bilayer PtSe $\mathrm{P}_{2}$. Blue and red arrows show the sites with missing Pt and Se atoms, respectively. Scale bar is $1 \mathrm{~nm}$. Bottom panel shows the intensity profile measured along the dashed orange line shown in the STEM image. Blue arrow shows the intensity recorded at the Pt vacancy site. c Top panel: device schematics. A few layer thick PtSe $\mathrm{C}_{2}$ with intrinsic defects is transferred on top of monolayer graphene. Both graphene and $\mathrm{PtSe}_{2}$ are contacted with non-magnetic Pd contacts (80-nm thick). Bottom panel: optical image of a completed device. Scale bar is $6 \mu \mathrm{m}$. d $V_{B G}$ dependence of $I_{D S}$ measured at fixed temperature values. $V_{D S}=2 \mathrm{~V}$. Inset shows the device schematic. e In $(G)$ vs $T^{-1 / 3}$ measured at different doping levels. Inset shows the temperature dependence of device conductance at different doping values. $\mathbf{f}$ Room-temperature $V_{B G}$ dependence of $I_{D S}$ measured at fixed $V_{D S}$ values. Inset compares the $V_{D S}$ dependence of measured off-state conductance for monolayer PtSe $e_{2}$ and $W S e_{2}$ prepared under the same conditions.
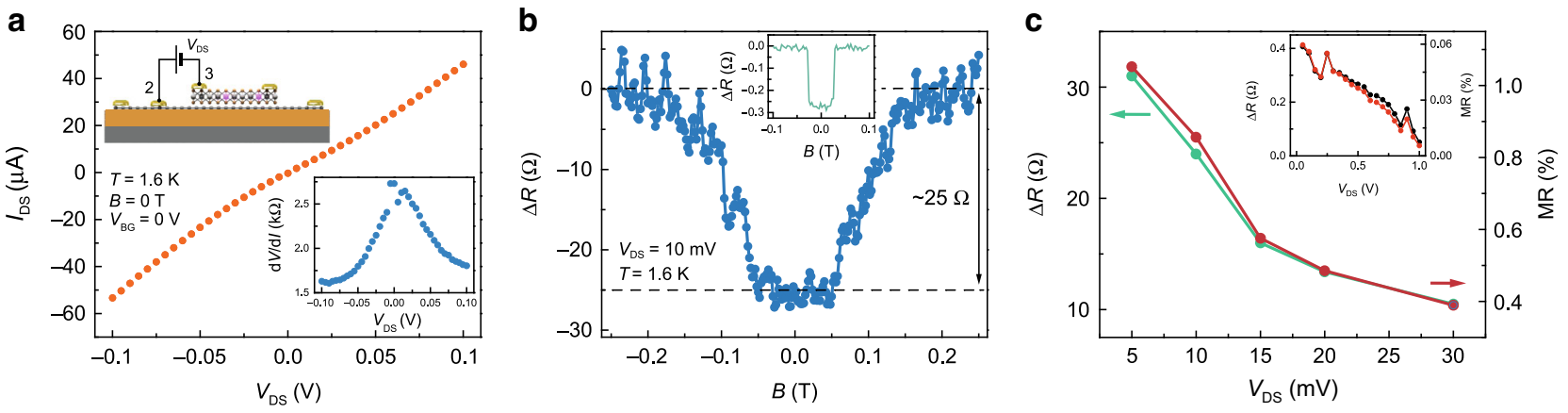

Fig. 2 Vertical transport across monolayer $\mathbf{P t S e}_{2}$. a Bias voltage dependence of $I_{D S}$. Top inset is the schematic of the measurement geometry. Bottom inset is the differential resistance as a function of applied bias. $\mathbf{b}$ Magnetic field dependence of electrical resistance measured at $T=1.6 \mathrm{~K}$. The observation of a two-plateau response indicates the presence of antiferromagnetic ground-state ordering. Inset shows the magneto-transport measurements for a 7nm-thick metallic $\mathrm{PtSe}_{2}$. c Bias dependence of the change in device resistance as a result of the magnetic field and corresponding MR percentage. All measurements are taken at $1.6 \mathrm{~K}$. Inset shows the bias dependence of resistance and MR for a $\sim 7$-nm-thick metallic PtSe $\mathrm{P}_{2}$. 
$V_{\mathrm{DS}}=10 \mathrm{mV}$. As clearly seen in Fig. $2 \mathrm{~b}$, the evolution of the resistance in our device with the magnetic field substantially departs from the typical localization response. Indeed, it features plateaus with two different values. As the field is increased, the lower plateau observed in the $-50 \mathrm{mT}<B<50 \mathrm{mT}$ interval is found to slowly approach the high plateau observed above 150 $\mathrm{mT}$. This two-plateau response is the signature of an antiferromagnetic ground-state ordering $20,30,31$. While we find a similar two-plateau response in thicker films of $\mathrm{PtSe}_{2}$ (inset of Fig. 2b), the observation of a slow low-to-high plateau switching in monolayer $\mathrm{PtSe}_{2}$ indicates that the magnetic moments are not fully oriented along the out-of-plane direction, with spins that could be slightly canted. Similarly to metallic films, hysteresis in monolayer $\mathrm{PtSe}_{2}$ is almost absent (see Supplementary Note 4). Furthermore, the magnetoresistance (MR) percentage is found to be bias-dependent as in thicker metallic devices and estimated to be $1 \%$ in the low-bias regime (Fig. $2 \mathrm{c}$ ).

In order to investigate the impact of magnetic ordering in $\mathrm{PtSe}_{2}$ on the transport properties of graphene, we characterize the graphene device resistance with and without $\mathrm{PtSe}_{2}$ on the channel connecting the source and drain electrodes. Figure 3 a displays the $V_{\mathrm{BG}}$ dependences of corresponding device resistances which show nearly identical device characteristics. However, the magnetic field responses of these junctions present striking differences, as shown in Fig. 3b, c. While the pristine graphene junction exhibits a conventional weak localization response ${ }^{32}$, the junction with the $\mathrm{PtSe}_{2}$ monolayer exhibits plateaus with two different values $^{20,30,31}$. This difference in the magneto-transport properties is clearly due to the presence of magnetism in $\mathrm{PtSe}_{2}$ and could be ascribed to two possible origins. One possibility is the back-andforth hopping of graphene's electrons through the magnetic defect states in $\mathrm{PtSe}_{2}$ via quantum tunneling, a mechanism similar
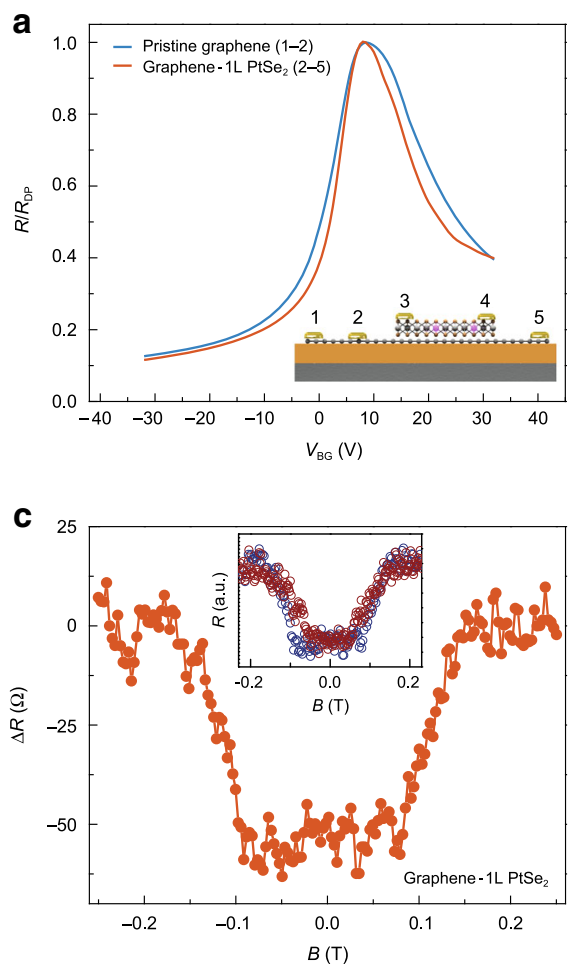

to the previously reported one in graphene/ $\mathrm{WS}_{2}$ heterostructures $^{33}$. This possibility is indeed supported by the observation of linear $I_{\mathrm{DS}}-V_{\mathrm{DS}}$ relation and the nearly identical shapes of the magnetic response of pure $\mathrm{PtSe}_{2}$ and the graphene/PtSe heterostructure, as shown in the inset of Fig. 3c. On the other hand, there could be contribution from the interface-induced magnetism ${ }^{34}$ by $\mathrm{PtSe}_{2}$.

Magnetoresistance measurement in bilayer $\mathrm{PtSe}_{2}$. Finally, we complement our investigation by replacing monolayer $\mathrm{PtSe}_{2}$ with bilayer $\mathrm{PtSe}_{2}$. We observe a hysteresis loop with minima at $\pm 15 \mathrm{mT}$ under backward and forward scans (Fig. $3 \mathrm{~d}$ ), which is the hallmark of ferromagnetism ${ }^{35}$. The maximum change in the device resistance upon the application of a magnetic field is $\sim 16 \Omega$ and it strongly depends on the applied $V_{\mathrm{DS}}$. The MR percentage probed by graphene is $\sim 0.1 \%$ and is nearly independent of the applied $V_{\mathrm{BG}}$ (see Supplementary Notes 5-6). Our findings suggest that monolayer (bilayer) $\mathrm{PtSe}_{2}$ exhibits long-range antiferromagnetic (ferromagnetic) ground-state ordering, and that adjacent graphene electrodes can effectively be used as a sensitive probe to detect such magnetism ${ }^{36}$. To shed more light on this antiferromagnetic-to-ferromagnetic crossover, measurement techniques such as single-spin microscopy ${ }^{37}$ can be employed at the nanoscale in future experiments. Such direct measurements would unambiguously determine the types of magnetism and its strength, as recently utilized for another layered magnet ${ }^{37}$.

Long-range magnetic ordering in semiconductors can be achieved through the incorporation of extrinsic magnetic impurities (e.g., Mn-doped $\mathrm{GaAs}^{38}$ and V-doped $\mathrm{WSe}_{2}{ }^{39}$ ). We rule out this possibility in our work by performing quantitative and qualitative assessment of our samples, which do not show the
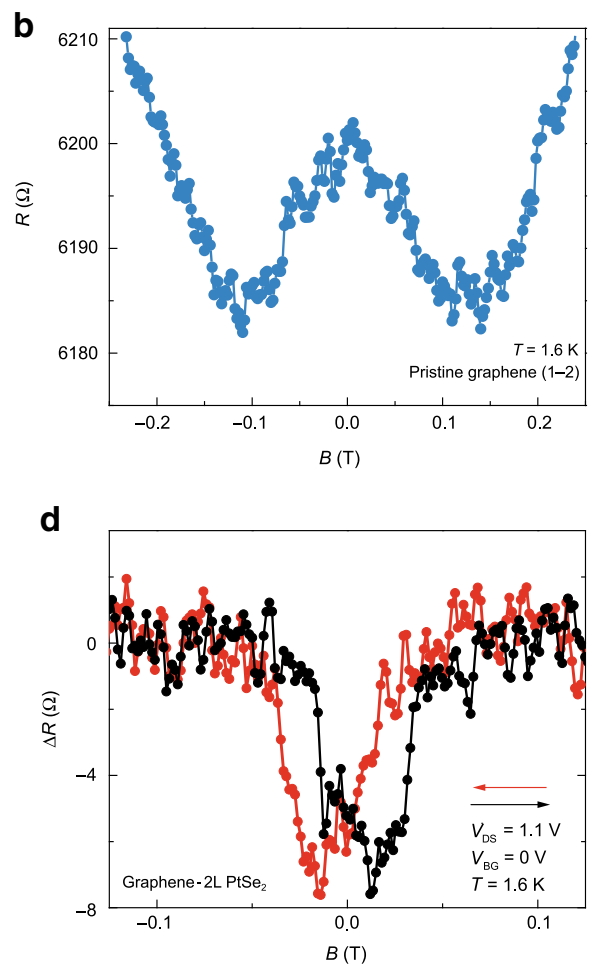

Fig. 3 Induced magnetization in monolayer graphene. a $V_{B G}$ dependence of graphene resistance. Red (blue) line represents the device with (without) a monolayer $\mathrm{PtSe}_{2}$ on top of the graphene channel, between the voltage probes. Inset is the schematics of the measurement geometry. $\mathbf{b}$ Magnetic field dependence of pristine graphene resistance measured at $T=1.6 \mathrm{~K}$. c Magnetic field dependence of graphene resistance measured at $T=1.6 \mathrm{~K}$ for the device with monolayer $\mathrm{PtSe}_{2}$ on the channel. Inset shows the magnetic field response of vertically measured monolayer PtSe ${ }_{2}$ (red circles) and laterally measured graphene/monolayer $\mathrm{PtSe}_{2}$ (blue circles). They have similar out-of-plane coercive fields. d Magnetic field dependence of graphene resistance measured at $T=1.6 \mathrm{~K}$ for the device with a bilayer $\mathrm{PtSe} \mathrm{e}_{2}$ on the graphene channel. Arrows represent magnetic field sweep directions. 
presence of any chemical elements except for Pt and Se and other crystal phases beside $1 \mathrm{~T}$ (see Supplementary Notes 7 and 8). Therefore, our findings constitute a model study demonstrating the purely defect-induced magnetism into an otherwise nonmagnetic crystal down to the ultimately scaled monolayer system.

First-principles calculations. We now address the physical mechanism underlying the observed effects from a theoretical perspective. According to a purely ionic argument, $\mathrm{Pt}$ atoms exhibit a formal oxidation state of +4 and a resulting valence electron configuration $5 \mathrm{~d}^{6} 6 \mathrm{~s}^{0}$. On the basis of the crystal and ligand fields associated with the octahedral environment in which each Pt atom resides, a splitting of the d orbitals into a pair of empty $e_{2 g}$ states and fully occupied $t_{2 g}$ states is expected. Such a full occupation of the $t_{2 g}$ manifold prevents any intrinsic magnetism in $\mathrm{PtSe}_{2}$ at all possible thicknesses, from monolayer to bulk, as further confirmed by our explicit density-functional calculations. As in the case of metallic $\mathrm{PtSe}_{2}{ }^{20}$, we conclude that lattice imperfections are the origin of the observed layerdependent magnetism in semiconducting $\mathrm{PtSe}_{2}$. Motivated by direct observation of $\mathrm{Pt}$ vacancies in our transmission electron microscope images shown in Fig. 1b, their indirect inference from our charge transport measurements as well as earlier scanning tunneling microscopy studies ${ }^{19}$, we investigate electronic and magnetic properties of mono- and bilayer models of $\mathrm{PtSe}_{2}$ with platinum vacancy defects by means of first-principles calculations. For the monolayer case, we find that a Pt vacancy defect in its fully spin-polarized configuration gives rise to a local magnetic moment of $4.00 \mu_{\mathrm{B}}$, which is localized mostly on the six selenium atoms neighboring to the missing $\mathrm{Pt}$ atom. We note, however, that the configuration characterized by local magnetic moments in the two Se atomic planes that are oriented antiparallel to each other (Fig. 4a) is $35 \mathrm{meV}$ lower in energy than the former parallel configuration. Below, we will refer to this configuration with zero net magnetic moment as antiferromagnetic. Here, we would like to also note that the Se point defect does not lead to magnetism.

Next, we consider the bilayer $\mathrm{PtSe}_{2}$ model with $\mathrm{Pt}$ vacancy in one of the two layers. In contrast to the monolayer case discussed above, we could not identify any solution for a defective bilayer other than the ferromagnetic configuration with a net magnetic moment of $1.33 \mu_{\mathrm{B}}$ (Fig. 4b). This value agrees well with the one obtained for Pt vacancy defects in thicker metallic $\mathrm{PtSe}_{2}$ slabs $^{20}$. The reason for this traces back to the existence of interlayer coupling between the $\mathrm{PtSe}_{2}$ sheets in the bilayer system. In our previous work ${ }^{20}$, we have shown that platinum vacancy defects located at the surfaces of multilayer $\mathrm{PtSe}_{2}$ act as magnetic centers, while their presence in the inner regions of the thick samples (as well as in the three-dimensional bulk material) does not lead to any local magnetic moment. This observation clearly pinpoints the pivotal role of interlayer interactions in quenching the defectinduced magnetic response in $\mathrm{PtSe}_{2}$. In bilayer $\mathrm{PtSe}_{2}$, the interlayer coupling affects only one of the two selenium atomic planes. As a result, the defect-induced magnetic moment in this system is reduced $\left(1.33 \mu_{\mathrm{B}}\right)$ as compared to the fully polarized magnetic moment of defect in monolayer $\mathrm{PtSe}_{2}\left(4.00 \mu_{\mathrm{B}}\right)$, and, remarkably, the spin density primarily resides on the outmost selenium atoms in the defect (Fig. 4b). Overall, our first-principles calculations suggest that a platinum vacancy defect yields an antiferro- (ferro-)magnetic ordering in mono (bi-)layer $\mathrm{PtSe}_{2}$, thereby portraying a theoretical picture in accord with our experimental observations.

\section{Discussion}

Antiferromagnets have been recently considered for sensing and memory applications ${ }^{40}$. For this purpose, layered intralayer
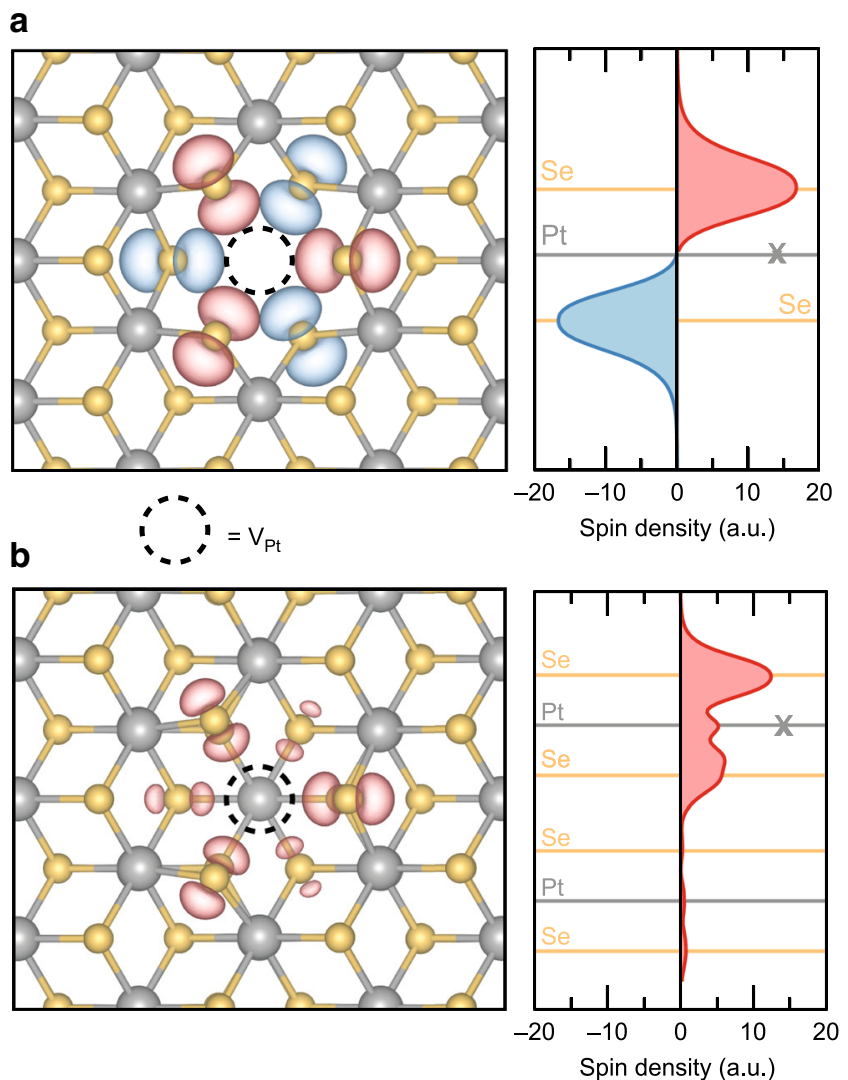

Fig. 4 Localized magnetic moments at Pt vacancy defects in $\mathbf{P t S e}_{\mathbf{2}}$. a Spin-density distribution at the ground-state configuration of the $\mathrm{V}_{\mathrm{Pt}}$ defect in monolayer $\mathrm{PtSe}_{2}$ calculated from first principles. Gray and orange balls represent Pt and Se atoms, respectively. The red and blue isosurfaces correspond to positive and negative spin densities. The position of the missing Pt atom is marked with a dashed circle. The right panel shows the in-plane averaged spin density as a function of the out-of-plane position. The cross $(X)$ indicates the Pt atomic plane at which the vacancy defect is introduced. b Spin-density distribution at the Pt vacancy in the top layer of bilayer $\mathrm{PtSe}_{2}$ calculated from first principles. Units in panels (a) and (b) agree with each other.

antiferromagnetic compounds such as $\mathrm{MnPS}_{3}, \mathrm{FePS}_{3}$, and $\mathrm{CoPS}_{3}$ indeed show promise 9 . Among these, only a few of them have been exfoliated down to the monolayer. While antiferromagnetic ordering at the monolayer limit still persists in $\mathrm{FePS}_{3}$ with an unchanged transition temperature ${ }^{41}$, such ordering is suppressed in $\mathrm{NiPS}_{3}{ }^{42}$. Unlike $\mathrm{FePS}_{3}$, the observed antiferromagnetic ordering in monolayer $\mathrm{PtSe}_{2}$ is not intrinsic, rather originates from native impurities hosted in the material. Therefore, our work ignites the potential of atomic-scale defects for driving nonmagnetic $2 \mathrm{D}$ crystals into magnetic phases, thereby opening new directions for expanding the library of two-dimensional magnets ${ }^{43}$.

\section{Methods}

Device fabrication. Our device fabrication starts with the mechanical exfoliation of monolayer graphene nanoribbons onto a doped Si substrate with $270 \mathrm{~nm}$ of $\mathrm{SiO}_{2}$. PtSe $e_{2}$ crystals are similarly obtained by the mechanical exfoliation from bulk crystals (HQ Graphene) onto a $\mathrm{Si} / \mathrm{SiO}_{2}$ wafer. The thickness of selected crystals was determined by using AFM topography imaging (Asylum Research Cypher). PtSe was then isolated from its $\mathrm{Si} / \mathrm{SiO}_{2}$ substrate with the help of a supporting PMMA layer by etching $\mathrm{SiO}_{2}$ via a diluted potassium hydroxide $(\mathrm{KOH})$ solution $(0.7 \%$, $\sim 3 \mathrm{~h}$ ). This was followed by rinsing the isolated film in deionized (DI) water for several times prior to its transfer onto previously exfoliated graphene nanoribbons. After removing the supporting PMMA via a hot acetone treatment $\left(\sim 90^{\circ} \mathrm{C}\right.$ for $\left.1 \mathrm{~h}\right)$ 
followed by cleaning with IPA. The resulting graphene/PtSe ${ }_{2}$ heterostructure was annealed at $250^{\circ} \mathrm{C}$ for $6 \mathrm{~h}$ under high vacuum conditions $\left(\sim 1 \times 10^{-6} \mathrm{mbar}\right)$ to further remove the transfer-related residues. Metallic contacts were prepared using e-beam lithography (Raith EBPG $5000+, 100 \mathrm{keV}$ thermal field emission gun with an e-beam dose of $\sim 950 \mu \mathrm{C} / \mathrm{cm}^{2}$ ) and e-beam evaporation of Pd (Alliance-Concept EVA $760,80-\mathrm{nm}$ thick). The deposition rate of Pd is $1 \AA / \mathrm{s}$ under the base pressure of $\sim 1 \times 10^{-6} \mathrm{mbar}$.

Transport measurements. Cryogenic measurements were performed in an ICE Oxford liquid helium continuous flow cryo-magnetic system with a base temperature of $\sim 1.5 \mathrm{~K}$. Source-drain currents were measured using a Keithley sourcemeter $2636 \mathrm{~B}$, while a Keithley sourcemeter 2400 was used for applying bias through the $\mathrm{SiO}_{2}$ gate dielectric. The source voltage was varying between 0 and $2 \mathrm{~V}$, while the drain was grounded.

Electron microscopy sample preparation and imaging. After the identification of 1-3 layers of $\mathrm{PtSe}_{2}$ on $\mathrm{Si} / \mathrm{SiO}_{2}$ substrate using AFM, the substrate was spin-coated with a PMMA A2 polymer (1500 rpm, 150-nm thick) and baked on a hot plate at $60^{\circ} \mathrm{C}$ for $4 \mathrm{~min}$. Then, PMMA surrounding the target $\mathrm{PtSe}_{2}$ was scratched and detached after mildly etching $\mathrm{SiO}_{2}$ using a dilute $\mathrm{KOH}$ solution $(0.7 \%, \sim 30 \mathrm{~min})$. The floating PMMA A2 film was picked up and rinsed several times in DI water. Finally, the film was aligned on $\mathrm{Si}_{3} \mathrm{~N}_{4}$ TEM grids with the help of a micromanipulator using a glass nanocapillary. Supporting PMMA film was removed by using the cleaning protocol discussed above. Resulting crystals on TEM grids were annealed at $250^{\circ} \mathrm{C}$ for $6 \mathrm{~h}$ under high vacuum conditions $\left(\sim 1 \times 10^{-6} \mathrm{mbar}\right)$ to further remove the transfer-related residues. Here, we note that a very diluted $\mathrm{KOH}$ concentration is used to eliminate the unintentional creation of defects in $\mathrm{PtSe}_{2}$. This effective transfer process was previously utilized for the STEM imaging of graphene, $\mathrm{MoS}_{2}$, and $\mathrm{WS}_{2}$ crystals which have extremely low concentration of defects.

Scanning transmission electron microscopy (STEM) imaging experiments were conducted using an aberration-corrected (with double Cs corrector) FEI Titan Themis TEM $60-300 \mathrm{kV}$, equipped with Schottky X-FEG electron source and a monochromator to reduce the effect of chromatic aberrations. To minimize the effect of electron-beam-induced knock-on damage, a low acceleration voltage $(80$ $\mathrm{kV}$ ) was used for all the experiments. The typical electron probe current was $20 \mathrm{pA}$. Images were acquired with Gatan high annular angular dark-field (HAADF) detector using $185-\mathrm{mm}$ camera length which corresponds to a $49.5-198 \mathrm{mrad}$ collection angle.

TEM simulations. Multislice STEM image simulations were performed using the Dr. Probe software package ${ }^{44}$. The frozen phonon method was used with 30 variations per pixels and 64 displacements per atom. The resulting sampling rate was $\sim 0.02 \mathrm{~nm} /$ pixel. The simulation parameters were chosen to be similar to the experimental conditions. The beam semi-convergence angle and HAADF detector angle was set to $21.2,49.5-198 \mathrm{mrad}$, respectively. The source size was chosen to $0.05 \mathrm{~nm}$ and all the aberrations were set to zero. The result of STEM image simulation confirms that there is no degradation or structural modification of $\mathrm{PtSe}_{2}$ in the process of transfer (see Supplementary Note 9).

First-principles calculations. Our calculations were performed under the gradientcorrected, spin-orbit inclusive density-functional theory formalism, as implemented in VASP ${ }^{45,46}$. We treated the exchange and correlation effects at the PBE level ${ }^{47}$, but additionally verified the robustness of our results against the choice of the density functional (see Supplementary Note 10). The energy cutoff for the plane wave basis set was set to $500 \mathrm{eV}$. The integration over the Brillouin zone was carried out with the equivalent of $12 \times 12 \times 1 \mathbf{k}$-points per unit cell. These parameters were chosen after extensive convergence tests. During the geometry optimizations, the in-plane lattice constants and interlayer spacing for the bilayer systems were constrained to their experimental values of 3.73 and $5.08 \AA$, respectively, while atomic coordinates were relaxed until each residual component on the Hellmann-Feynman forces was lower than $0.01 \mathrm{eV}^{-1}$. We modeled defective mono and bilayer PtSe $e_{2}$ by introducing a single Pt vacancy defect in an otherwise pristine $4 \times 4$ supercell containing 48 and 96 atoms, respectively, as such models host a Pt vacancy concentration of $14 \times 10^{12} \mathrm{~cm}^{-2}$, very close to our experimental estimate of $\sim 9 \times 10^{12} \mathrm{~cm}^{-2}$. In Supplementary Note 10 , we additionally show that our conclusions are insensitive to the size of the supercell. A vacuum region of $15 \AA$ was introduced to separate the periodic images.

\section{Data availability}

The data that support the findings of this study are available from the corresponding author on reasonable request.

Received: 22 January 2020; Accepted: 21 August 2020; Published online: 23 September 2020

\section{References}

1. Manzeli, S., Ovchinnikov, D., Pasquier, D., Yazyev, O. V. \& Kis, A. 2D transition metal dichalcogenides. Nat. Rev. Mater. 2, 17033 (2017).

2. Radisavljevic, B., Radenovic, A., Brivio, J., Giacometti, V. \& Kis, A. Single-layer $\mathrm{MoS}_{2}$ transistors. Nat. Nanotechnol. 6, 147-150 (2011).

3. Žutić, I., Fabian, J. \& Das Sarma, S. Spintronics: fundamentals and applications. Rev. Mod. Phys. 76, 323-410 (2004).

4. Han, W., Kawakami, R. K., Gmitra, M. \& Fabian, J. Graphene spintronics. Nat. Nanotechnol. 9, 794-807 (2014)

5. Lin, X., Yang, W., Wang, K. L. \& Zhao, W. Two-dimensional spintronics for low-power electronics. Nat. Electron 2, 274-283 (2019).

6. Avsar, A. et al. Colloquium: spintronics in graphene and other twodimensional materials. Rev. Mod. Phys. 92, 021003 (2020).

7. Burch, K. S., Mandrus, D. \& Park, J.-G. Magnetism in two-dimensional van der Waals materials. Nature 563, 47-52 (2018).

8. Gong, C. \& Zhang, X. Two-dimensional magnetic crystals and emergent heterostructure devices. Science 363, eaav4450 (2019).

9. Mak, K. F., Shan, J. \& Ralph, D. C. Probing and controlling magnetic states in 2D layered magnetic materials. Nat. Rev. Phys. 1, 646-661 (2019).

10. Gibertini, M., Koperski, M., Morpurgo, A. F. \& Novoselov, K. S. Magnetic 2D materials and heterostructures. Nat. Nanotechnol. 14, 408-419 (2019).

11. Ciarrocchi, A., Avsar, A., Ovchinnikov, D. \& Kis, A. Thickness-modulated metal-to-semiconductor transformation in a transition metal dichalcogenide. Nat. Commun. 9, 919 (2018).

12. Wang, Y. et al. Monolayer $\mathrm{PtSe}_{2}$, a new semiconducting transition-metaldichalcogenide, epitaxially grown by direct selenization of Pt. Nano Lett. 15, 4013-4018 (2015).

13. Zhao, Y. et al. High-electron-mobility and air-stable $2 \mathrm{D}$ layered $\mathrm{PtSe}_{2}$ FETs. Adv. Mater. 29, 1604230 (2017).

14. Yim, C. et al. High-performance hybrid electronic devices from layered $\mathrm{PtSe}_{2}$ films grown at low temperature. ACS Nano 10, 9550-9558 (2016).

15. Yim, C. et al. Electrical devices from top-down structured platinum diselenide films. npj 2D Mater. Appl. 2, 5 (2018).

16. Miró, P., Ghorbani-Asl, M. \& Heine, T. Two dimensional materials beyond $\mathrm{MoS}_{2}$ : noble-transition-metal dichalcogenides. Angew. Chem. Int. Ed. 53, 3015-3018 (2014)

17. Kurpas, M. \& Fabian, J. Intrinsic and extrinsic spin-orbit coupling and spin relaxation in monolayer $\mathrm{PtSe}_{2}$. https://arxiv.org/abs/2006.03384 (2020).

18. Gao, J. et al. Structure, stability, and kinetics of vacancy defects in monolayer $\mathrm{PtSe}_{2}$ : a first-principles study. ACS Omega 2, 8640-8648 (2017).

19. Zheng, H. et al. Visualization of point defects in ultrathin layered $1 \mathrm{~T}-\mathrm{PtSe}_{2} .2 \mathrm{D}$ Mater. 6, 041005 (2019).

20. Avsar, A. et al. Defect induced, layer-modulated magnetism in ultrathin metallic $\mathrm{PtSe}_{2}$. Nat. Nanotechnol. 14, 674-678 (2019).

21. Guguchia et al. Magnetism in semiconducting molybdenum dichalcogenides. Sci. Adv. 4, eaat3672 (2018).

22. Furdyna, J. K. Diluted magnetic semiconductors. J. Appl. Phys. 64, R29 (1988).

23. Avsar, A. et al. Reconfigurable diodes based on vertical $\mathrm{WSe}_{2}$ transistors with van der Waals bonded contacts. Adv. Mater. 30, 1707200 (2018).

24. Mott, N. F. Conduction in non-crystalline materials. Philos. Mag. 19, 835-852 (1969).

25. Han, M. Y., Brant, J. C. \& Kim, P. Electron transport in disordered graphene nanoribbons. Phys. Rev. Lett. 104, 056801 (2010).

26. Radisavljevic, B. \& Kis, A. Mobility engineering and a metal-insulator transition in monolayer $\mathrm{MoS}_{2}$. Nat. Mater. 12, 815-820 (2013).

27. Qiu, H. et al. Hopping transport through defect-induced localized states in molybdenum disulphide. Nat. Commun. 4, 2642 (2013).

28. Cao, Y. et al. Quality heterostructures from two-dimensional crystals unstable in air by their assembly in inert atmosphere. Nano Lett. 15, 4914-4921 (2015).

29. Pradhan, N. R. et al. Ambipolar molybdenum diselenide field-effect transistors: field-effect and hall mobilities. ACS Nano 8, 7923-7929 (2014).

30. Klein, D. R. et al. Probing magnetism in $2 \mathrm{D}$ van der Waals crystalline insulators via electron tunneling. Science 360, 1218-1222 (2018).

31. Song, T. et al. Giant tunneling magnetoresistance in spin-filter van der Waals heterostructures. Science 360, 1214-1218 (2018).

32. Tikhonenko, F. V., Horsell, D. W., Gorbachev, R. V. \& Savchenko, A. K. Weak localization in graphene flakes. Phys. Rev. Lett. 100, 056802 (2008).

33. Avsar, A. et al. Spin-orbit proximity effect in graphene. Nat. Commun. 5, 4875 (2014).

34. Žutić, I., Matos-Abiague, A., Scharf, B., Dery, H. \& Belashchenko, K. Proximitized materials. Mater. Today 22, 85-107 (2019).

35. Leven, B. \& Dumpich, G. Resistance behavior and magnetization reversal analysis of individual Co nanowires. Phys. Rev. B 71, 064411 (2005).

36. Kim, M. et al. Micromagnetometry of two-dimensional ferromagnets. Nat. Electron 2, 457-463 (2019).

37. Thiel, L. et al. Probing magnetism in $2 \mathrm{D}$ materials at the nanoscale with single-spin microscopy. Science 364, 973-976 (2019). 
38. Dietl, T. \& Ohno, H. Dilute ferromagnetic semiconductors: physics and spintronic structures. Rev. Mod. Phys. 86, 187-251 (2014).

39. Yun, S. J. et al. Ferromagnetic order at room temperature in monolayer $\mathrm{WSe}_{2}$ semiconductor via vanadium dopant. Adv. Sci. 7, 1903076 (2020).

40. Jungwirth, T. et al. The multiple directions of antiferromagnetic spintronics. Nat. Phys. 14, 200-203 (2018).

41. Lee, J.-U. et al. Ising-type magnetic ordering in atomically thin $\mathrm{FePS}_{3}$. Nano Lett. 16, 7433-7438 (2016).

42. Kim, K. et al. Suppression of magnetic ordering in XXZ-type antiferromagnetic monolayer $\mathrm{NiPS}_{3}$. Nat. Commun. 10, 345 (2019).

43. Cortie, D. L. et al. Two-dimensional magnets: forgotten history and recent progress towards spintronic applications. Adv. Funct. Mater. 30, 1901414 (2019).

44. Barthel, J. Dr. Probe: a software for high-resolution STEM image simulation. Ultramicroscopy 193, 1-11 (2018).

45. Kresse, G. \& Joubert, D. From ultrasoft pseudopotentials to the projector augmented-wave method. Phys. Rev. B 59, 1758-1775 (1999).

46. Kresse, G. \& Furthmüller, J. Efficient iterative schemes for ab initio totalenergy calculations using a plane-wave basis set. Phys. Rev. B 54, 11169-11186 (1996).

47. Perdew, J. P., Burke, K. \& Ernzerhof, M. Generalized gradient approximation made simple. Phys. Rev. Lett. 77, 3865-3868 (1996).

\section{Acknowledgements}

We acknowledge the help of M. Lihter and A. Radenovic with sharing their recipe for the transfer of exfoliated 2D materials on TEM grids. We also acknowledge the help of N. Gasilova (ISIC) with ICP-MS characterization of $\mathrm{PtSe}_{2}$. We thank Z. Benes (CMI) for the help with electron-beam lithography and the Centre Interdisciplinaire de Microscopie Electronique (CIME) at EPFL for access to electron microscopes as well as R. Zamani for technical assistance with TEM imaging. A.A., C-Y.C., M.T., A.C., and A.K. would like to acknowledge support by the European Research Council (ERC, Grant 682332), Swiss National Science Foundation (Grant 175822), and Marie Curie-Sklodowska COFUND (grant 665667). A.K. acknowledges funding from the European Union's Horizon 2020 research and innovation program under grant agreements No. 785219 and 881603 (Graphene Flagship). M.P. and O.V.Y. acknowledge support by the Swiss National Science Foundation (Grant 172543). First-principles calculations have been carried out at the Swiss National Supercomputing Centre (CSCS) under the projects s832 and s1008.

\section{Author contributions}

A.A. and A.K. designed the experiments. A.A., C-Y.C., and A.C. fabricated the samples and performed transport measurements. M.T. performed TEM imaging and simulations. M.P. and O.V.Y. devised models and performed first-principles calculations. A.A., M.P., and A.K. wrote the paper with input from all authors.

\section{Competing interests}

The authors declare no competing interests.

\section{Additional information}

Supplementary information is available for this paper at https://doi.org/10.1038/s41467020-18521-6.

Correspondence and requests for materials should be addressed to A.A. or A.K.

Peer review information Nature Communications thanks Guido Menichetti and the other, anonymous, reviewer(s) for their contribution to the peer review of this work

Reprints and permission information is available at http://www.nature.com/reprints

Publisher's note Springer Nature remains neutral with regard to jurisdictional claims in published maps and institutional affiliations.

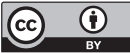

Open Access This article is licensed under a Creative Commons Attribution 4.0 International License, which permits use, sharing, adaptation, distribution and reproduction in any medium or format, as long as you give appropriate credit to the original author(s) and the source, provide a link to the Creative Commons license, and indicate if changes were made. The images or other third party material in this article are included in the article's Creative Commons license, unless indicated otherwise in a credit line to the material. If material is not included in the article's Creative Commons license and your intended use is not permitted by statutory regulation or exceeds the permitted use, you will need to obtain permission directly from the copyright holder. To view a copy of this license, visit http://creativecommons.org/ licenses/by/4.0/

(C) The Author(s) 2020 